\title{
Prolonged inhibition and incomplete recovery of mitochondrial function in oxazolidinone-treated megakaryoblastic cell lines
}

\author{
Tamara V. Milosevic a, Gaëlle Vertenoeil ${ }^{\mathrm{b}, \mathrm{c}}$, Valéry L. Payen ${ }^{\mathrm{d}, \dagger}$, Pierre Sonveaux ${ }^{\mathrm{d}}$, \\ Paul M. Tulkens ${ }^{a}$, Stefan N. Constantinescu ${ }^{\mathrm{b}, c, e}$, Françoise Van Bambeke ${ }^{\mathrm{a}, *}$ \\ a Pharmacologie cellulaire et moléculaire, Louvain Drug Research Institute, Université catholique de Louvain, Brussels, Belgium \\ b Signal Transduction and Molecular Haematology, Ludwig Institute for Cancer Research, Brussels Branch, Brussels, Belgium \\ ' de Duve Institute, Université catholique de Louvain, Brussels, Belgium \\ d Pole of Pharmacology and Therapeutics, Institute of Experimental and Clinical Research, Université catholique de Louvain, Brussels, Belgium \\ e Walloon Excellence in Life Science and Biotechnology, Brussels, Belgium
}

\section{A R T I C L E I N F O}

\section{Article history:}

Received 27 May 2019

Accepted 21 July 2019

Editor: Dr. Jim Gray

\section{Keywords:}

Oxazolidinone

Megakaryocyte

Megakaryoblast

Mitochondria

Cytochrome c-oxidase

Thrombocytopenia

\begin{abstract}
A B S T R A C T
Thrombocytopenia is commonly seen in patients receiving linezolid for $>14$ days. Linezolid is a reversible inhibitor of mitochondrial function in various cell types. This study investigated the inhibitory effects of linezolid and tedizolid, and their potential recovery on (i) CYTox I expression (subunit I of cytochrome coxidase; encoded by the mitochondrial genome), (ii) cytochrome c-oxidase activity and (iii) mitochondrial respiration (Seahorse bioanalysis) in two megakaryocytic cell lines [UT-7 WT (human acute megakaryoblastic leukaemia cells) and UT-7 MPL (transduced to express the thrombopoietin receptor)]. Cells were exposed to linezolid $(0.5-25 \mathrm{mg} / \mathrm{L})$ or tedizolid $(0.1-5 \mathrm{mg} / \mathrm{L})$ for up to 5 days and recovery followed after drug removal. Both oxazolidinones caused concentration- and time-dependent inhibition of CYTox I expression, cytochrome $c$-oxidase activity and mitochondrial spare capacity. On electron microscopy, mitochondria appeared dilated with a loss of cristae. Globally, tedizolid exerted stronger effects than linezolid. While CYTox I expression recovered completely after 6 days of drug washout, only partial (linezolid) or no (tedizolid) recovery of cytochrome c-oxidase activity, and no rescue of mitochondrial spare capacity (after 3 days) was observed. Thus, and in contrast to previous studies using a variety of cell lines unrelated to megakaryocytic lineages, the inhibitory effects exerted by oxazolidinones on the mitochondrial function of megakaryoblastic cells appear to be particularly protracted. Given the dynamics of platelet production and destruction, these results may explain why oxazolidinone-induced thrombocytopenia is one of the most common side effects in patients exposed to these antibiotics.
\end{abstract}

(c) 2019 Elsevier B.V. and International Society of Chemotherapy. All rights reserved.

\section{Introduction}

Oxazolidinones are the latest class of antibiotics with a novel mode of action to reach widespread clinical use. They inhibit bacterial protein synthesis by binding to the ribosomal $50 \mathrm{~S}$ subunit and impairing formation of the initiation complex [1]. They are active against most multi-resistant staphylococci and enterococci of clinical origin [2]. Due to their excellent oral bioavailability,

\footnotetext{
* Corresponding author at: Pharmacologie cellulaire et moléculaire, Louvain Drug Research Institute, avenue E. Mounier 73 Bte B1.73.05, B-1200 Bruxelles, Belgium. Tel.: +32 27647378 .

E-mail addresses: tulkens@facm.ucl.ac.be (P.M. Tulkens), francoise.vanbambeke@uclouvain.be (F. Van Bambeke).

$\dagger$ Current affiliation: Institute of Experimental and Clinical Research, Paediatrics Pole, and Louvain Drug Research Institute, Advanced Drug Delivery and Biomaterials, Université catholique de Louvain, Brussels, Belgium.
}

they are often selected for early discharge of patients. Unfortunately, treatment with linezolid (the first approved oxazolidinone) for $>14$ days and/or in patients with renal insufficiency is frequently associated with haematological side effects, with thrombocytopenia affecting up to $30 \%$ of patients [3-5]. Although approximately two- to eight-fold more potent than linezolid [2], tedizolid was reported to cause less thrombocytopenia based on the results of dose-escalating phase I trials and of fixed-dose, 6-day-treatment phase III clinical trials [6]. However, this has been challenged based on data from the Food and Drug Administration registry covering 18 months clinical use of tedizolid [7].

Given the similarity between bacterial and mitochondrial ribosomes, oxazolidinones also bind to mitochondrial ribosomes, impair the expression and synthesis of mitochondrial proteins encoded by the mitochondrial genome [8,9], and inhibit the activity of cytochrome $c$-oxidase and mitochondrial oxidative activity [9]. These effects are rationalized by structural similarities between 
a
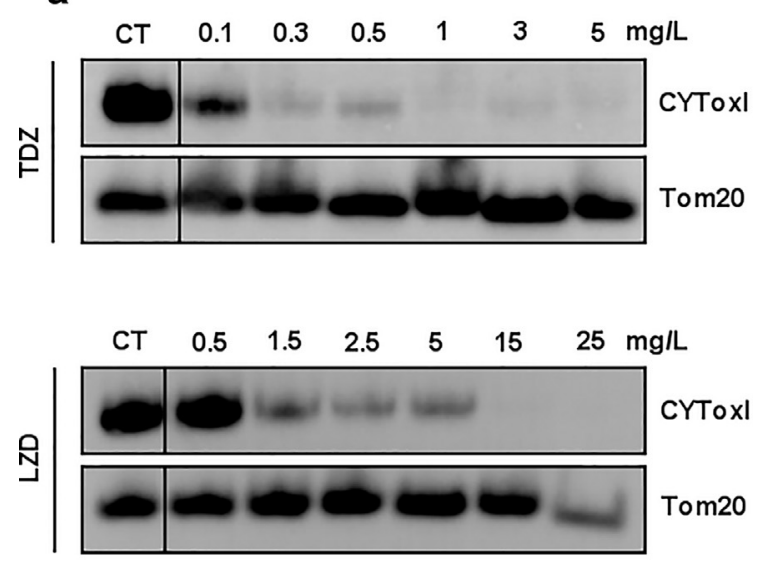

UT-7 MPL
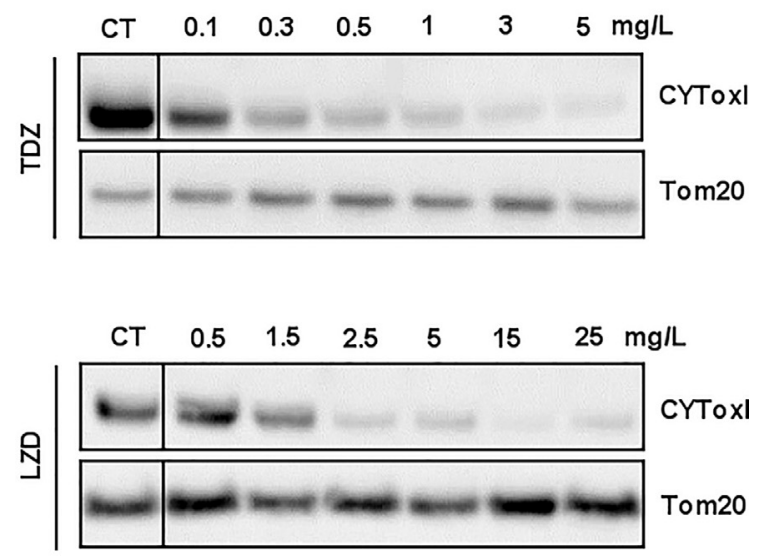

b
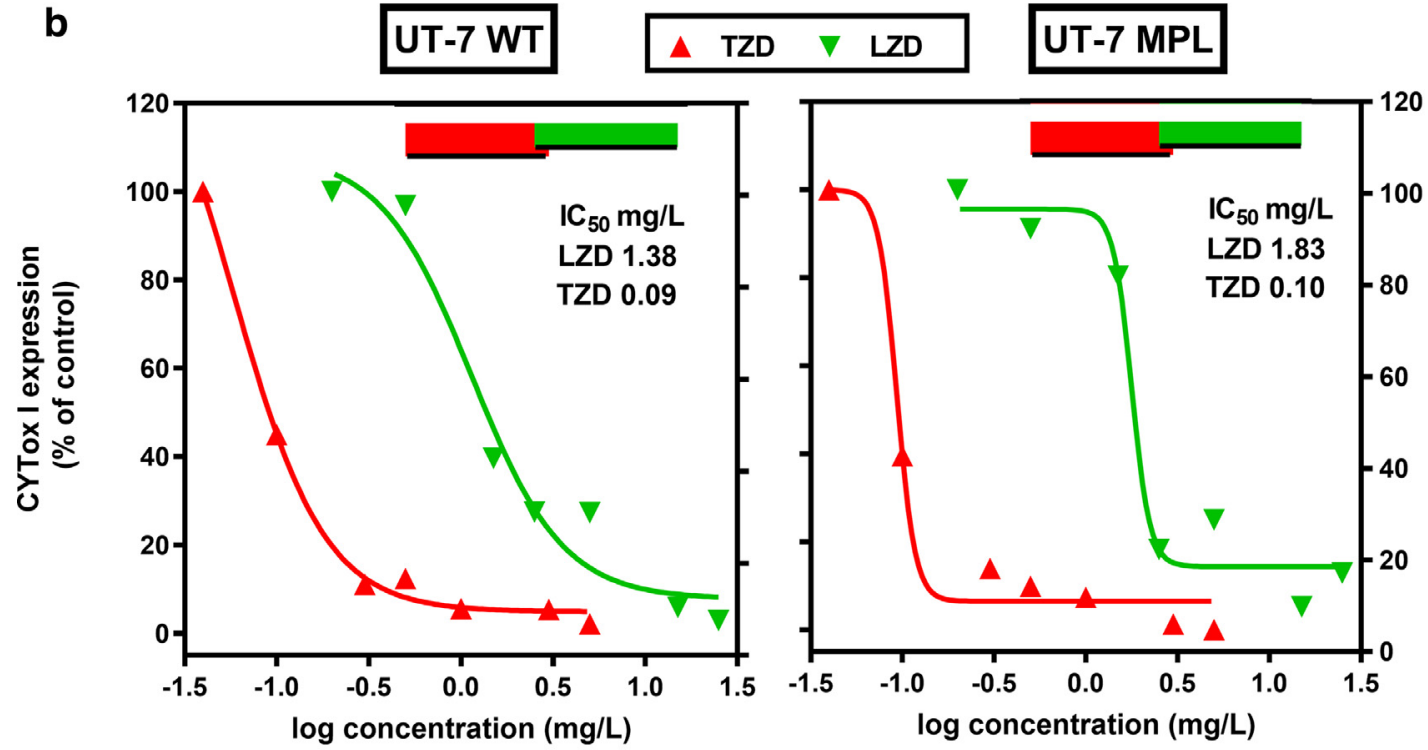

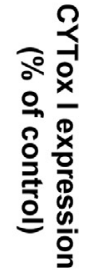
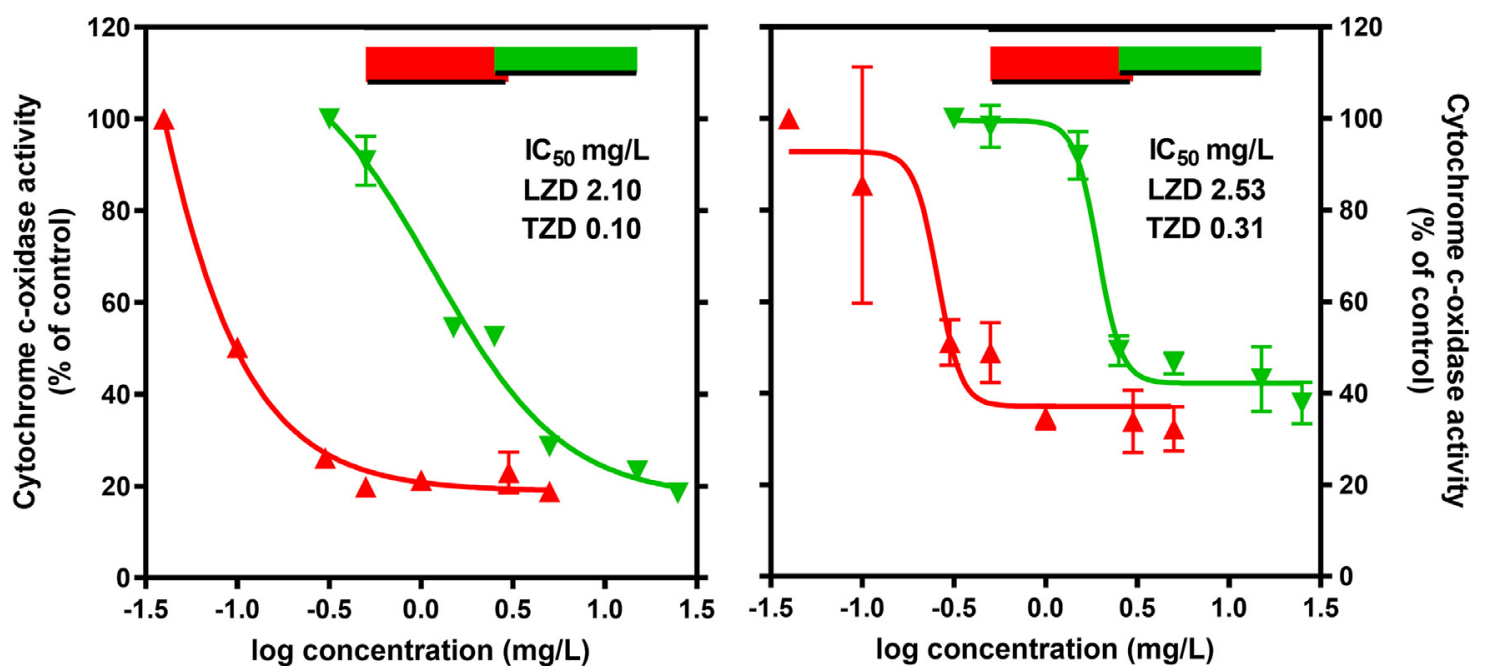

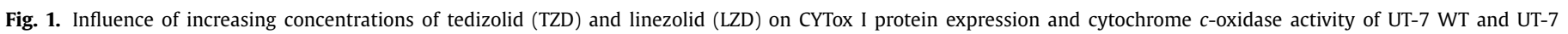

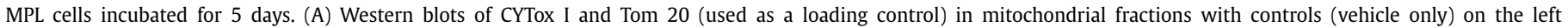

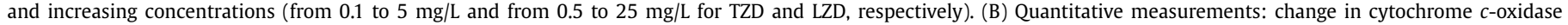

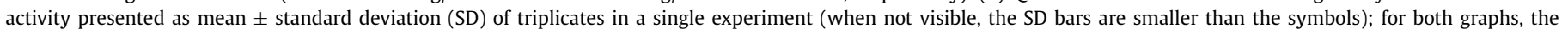

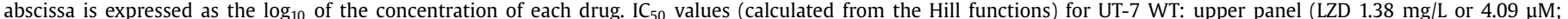

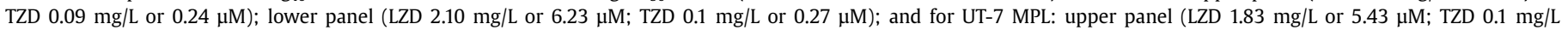

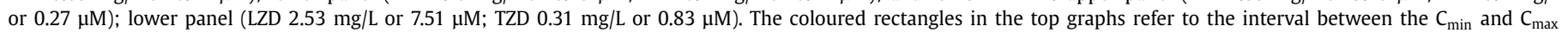
values commonly observed in serum of humans $(0.5-3 \mathrm{mg} / \mathrm{L}$ and $2-15 \mathrm{mg} / \mathrm{L}$, respectively) receiving conventional doses of TZD (200 mg) or LZD (600 mg). 
bacterial and mitochondrial ribosomes, with common binding sites for oxazolidinones [10]. Most notably, tedizolid possesses additional interactions with the oxazolidinone target site in bacterial ribosomes [11]. It also causes higher inhibition than linezolid for (i) mitochondrial protein synthesis in isolated cardiac mitochondria [12], and (ii) CYTox I expression (a protein encoded by the mitochondrial genome) and cytochrome c-oxidase activity in human HL-60 promyelocytes and THP-1 monocytes [9]. These cell lines, however, have no or only a weak link to the megakaryocytic lineage, drawing into question their predictive value to assess the impact of oxazolidinones on platelet precursors. In this context, this study examined the impact of linezolid and tedizolid on the expression of CYTox I and on cytochrome c-oxidase activity in UT-7 cells, considered as a useful in-vitro surrogate of megakaryoblasts. They were compared with UT-7 cells transduced to express a functional thrombopoietin (TPO) receptor, corresponding to more differentiated megakaryocytes.

\section{Methods}

Linezolid (potency 99.2\%) was obtained as RX-0366-00-005 from Rib-X Pharmaceuticals (presently Melinta Therapeutics, New Haven, CT, USA), and tedizolid (potency 101.4\%) as microbiological standard from Trius Pharmaceuticals (San Diego, CA, USA) and thereafter from Cubist Pharmaceuticals GmbH (Zürich, Switzerland), both now parts of Merck \& Co. (Kenilworth, NJ, USA). Stock solutions were prepared in DMSO, and diluted to bring the final DMSO concentration in the culture fluid to $0.5 \%$ (no cytotoxicity observed). Granulocyte-macrophage colony-stimulating factor (GM-CSF) and human TPO were obtained from Miltenyi Biotec $\mathrm{GmbH}$, Bergisch Gladbach, Germany.

UT-7 WT human acute megakaryoblastic leukaemia cells were obtained from the German Collection of Microorganisms and Cell Cultures (Deutsche Sammlung von Mikroorganismen und Zellkulturen, Braunschweig, Germany) [13]. UT-7 MPL cells [14] are UT-7 WT cells transduced with viral particles derived from packaging cell lines transfected with pMex-ires-GFP HA huMPL vector that code for the human TPO receptor (TpoR or c-MPL). These cells were grown in MEM-alpha GlutaMAX medium (Gibco-Life Technologies, Thermo-Fisher Scientific Inc., Waltham, MA, USA) supplemented with $10 \%$ fetal bovine serum (Gibco-Life Technologies) and GM-CSF at $10 \mathrm{ng} / \mathrm{mL}$ for UT-7 WT, or TPO at $10 \mathrm{ng} / \mathrm{mL}$ for UT-7 MPL. They were incubated at $37^{\circ} \mathrm{C}$ in a $5 \% \mathrm{CO}_{2}$-air atmosphere. Proliferation was evaluated using an automated cell counter (Beckman Coulter Life Science, Indianapolis, IN, USA).

Mitochondria-enriched preparations were obtained as described previously [9]. Protein concentrations were measured using a bicinchoninic acid assay (BCA Protein Assay Reagent, Pierce, Thermo Fisher, Waltham, MA, USA), with equal amounts of proteins separated by NuPAGE using $10 \%$ Bis-Tris precasted gels. The following primary antibodies were used: mouse anti-cytochrome c-oxidase subunit I (CYTox I) monoclonal antibody (Anti-OxPhos Complex IV Subunit I Monoclonal Antibody; Invitrogen cat. no. 459600 ) at $0.4 \mu \mathrm{g} / \mathrm{mL}$ and rabbit anti-Tom 20 polyclonal antibody (Invitrogen cat. no. PA5-39247) at $0.2 \mu \mathrm{g} / \mathrm{mL}$. Secondary antibodies (rabbit anti-mouse IgG (Invitrogen cat. no. A27025) and goat anti-rabbit IgG antibody (Invitrogen cat. no. A27036) were used at a concentration of $0.4 \mu \mathrm{g} / \mathrm{mL}$. Band intensity was quantified on membranes scanned using Chemiluminescence Imaging-Fusion PULSE apparatus (Analis, Namur, Belgium) with Image J software (National Institutes of Health, Bethesda, MD, USA; available from https://imagej.nih.gov/ij/).

Cytochrome c-oxidase activity was assayed on sonicated cells as described previously [9] after exposure to $0.2 \%$ digitonin, and calculated as the slope of the change in absorption of reduced cytochrome $c$ over time after logarithmic linearization, normalized based on the protein content of the cell lysate, determined by Lowry's method.

For mitochondrial oxygen consumption rate measurements, cells were collected by low speed centrifugation, and resuspended at a suitable dilution in Dulbecco's modified Eagle's medium (Sigma-Aldrich cat. no. D5030, Sigma Aldrich, St Louis, MO, USA) supplemented with $1.85 \mathrm{~g} / \mathrm{L} \mathrm{NaCl}, 10 \mathrm{mM}$ D-glucose and $2 \mathrm{mM}$ L-glutamine, and seeded in a poly-L-lysine-coated (Sigma-Aldrich cat. no. P6282) Seahorse XF96 V3 PS cell culture microplate (Agilent cat. no. 101085-004, Agilent Technologies, Santa Clara, CA, USA) at densities of 250,000 cells/well. Oxygen consumption rate (OCR) and its various stages (basal respiration, ATP-linked respiration, spare capacity and maximal respiration) were measured on a Seahorse XF96 analyzer as described in [9]. Data were normalized by cell count using a SpectraMax i3 plate imager (Molecular Devices, Sunnyvale, CA, USA) and SoftMax Pro software (Molecular Devices).

Preparation of samples for electron microscopy was performed as described previously [9].

Products not described above were obtained from SigmaAldrich or Merck KGaA (Darmstadt, Germany).

Curve fitting and statistical analyses were performed using GraphPad Prism Version 8.1.1. (330) and GraphPad InStat Version 3.10 for Windows (GraphPad Software, Inc., San Diego, CA, USA, www.graphpad.com).

\section{Results}

\subsection{Oxazolidinones at increasing concentrations inhibit CYTox I expression and cytochrome c-oxidase activity}

CYTox I (one of 13 proteins of the mitochondrial respiratory chain encoded by the mitochondrial genome) expression was examined in UT-7 WT and UT-7 MPL megakaryocytic cell lines incubated for 5 days with linezolid or tedizolid over a wide range of concentrations chosen to cover that of clinically relevant concentrations (see Fig. 1). The expression of Tom20, a translocase from the outer mitochondrial membrane encoded by the nuclear genome, was measured in parallel. While the expression of Tom20 was not affected by oxazolidinones, both drugs inhibited CYTox I expression in a concentration-dependent manner. The protein became almost undetectable for concentrations of linezolid $>15 \mathrm{mg} / \mathrm{L}$ and of tedizolid of $0.3 \mathrm{mg} / \mathrm{L}$ (Fig. 1A). Calculations of the concentration of antibiotic causing $50 \%$ activity inhibition $\left(\mathrm{IC}_{50}\right)$ showed that tedizolid was 17-20 times more potent as an inhibitor than linezolid in UT-7 WT and UT-7 MPL cells, respectively [Fig. 1B (upper panel); see caption for IC $_{50}$ values].

The enzymatic activity of cytochrome $c$-oxidase was measured in parallel in the same conditions [Fig. 1B (lower panel)]. Both oxazolidinones induced a concentration-dependent decrease in enzymatic activity, with tedizolid being more potent than linezolid ( 9 to 24 times in UT-7 MPL and UT-7 WT, respectively).

UT-7 WT cells were therefore slightly more susceptible to linezolid treatment than UT-7 MPL cells (1.3-fold decrease in CYTox I protein expression and 1.2-fold decrease in enzyme activity). For tedizolid, the ratio of $\mathrm{IC}_{50}$ values for UT-7 WT to UT-7 MPL was approximately 1 for CYTox I expression but 3 for enzyme activity.

3.2. Influence of the time of incubation with oxazolidinones on CYTox I protein expression, cytochrome c-oxidase activity, mitochondrial respiration and ultrastructure

Changes in CYTox I protein expression were followed over time in cells exposed to a fixed concentration of both drugs corresponding to their human $\mathrm{C}_{\max }$ (linezolid: $15 \mathrm{mg} / \mathrm{L}$; tedizolid: 
$3 \mathrm{mg} / \mathrm{L})$. Data are presented in Fig. 2A,B. Both drugs completely inhibited CYTox I protein expression after $72 \mathrm{~h}$ in UT-7 WT cells, as well as linezolid in UT-7 MPL cells. However, a residual activity of approximately $20 \%$ was observed in UT-7 MPL cells treated with tedizolid. In order to assess the potential functional impact of this decrease, cytochrome c-oxidase activity and mitochondrial respiration of UT-7 WT and UT-7 MPL cells were measured (Fig. 2C,D). Cells were incubated for $72 \mathrm{~h}$ at a concentration corresponding to the $C_{\max }$ of oxazolidinones, at which maximal inhibition of CYTox I expression was obtained. As shown in Fig. 2C, enzyme activity was significantly reduced to approximately 50$75 \%$ in both cell lines. Fig. 2D shows that basal mitochondrial OCR and spare capacity (i.e. the ability to increase mitochondrial OCR from a basal to a maximal level) were reduced by either oxazolidinone in UT-7 WT cells, while only spare capacity was impaired in UT-7 MPL cells. Typical images of mitochondria under control conditions and after incubation with oxazolidinones at their $\mathrm{C}_{\max }$ for $72 \mathrm{~h}$ are shown in Fig. 2E. Mitochondria appeared less dense in UT-7 MPL cells than in their WT counterparts, with fewer cristae. In oxazolidinone-exposed cells, mitochondria were dilated in both cell types and harboured scarce, disorganized cristae.

\subsection{Reversibility of the inhibition of CYTox I expression, cytochrome c-oxidase activity and mitochondrial spare capacity}

Cells were treated for $72 \mathrm{~h}$ with oxazolidinones at their $\mathrm{C}_{\max }$ (to obtain maximal inhibition of cytochrome $c$-oxidase subunit I
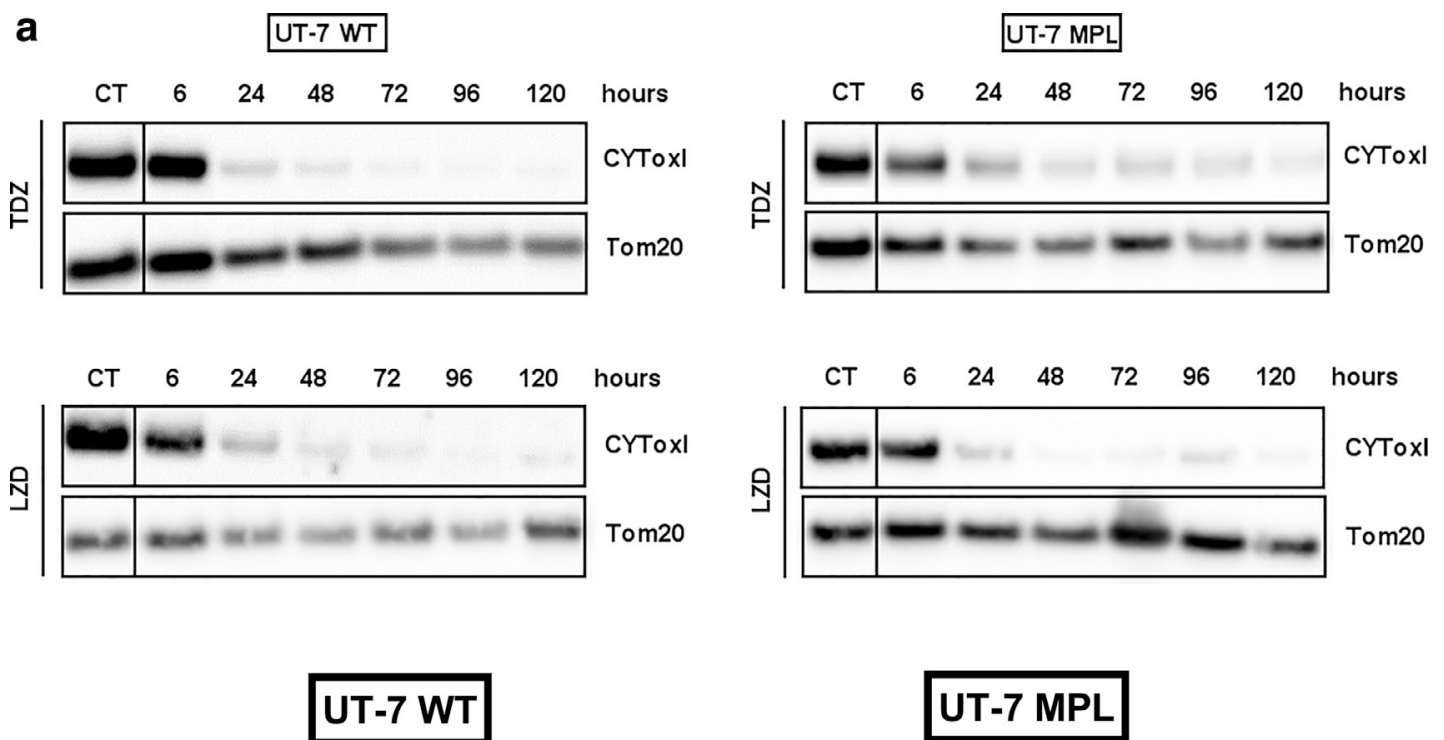

UT-7 MPL

\section{B. cytochrome c-oxidase subunit I expression}
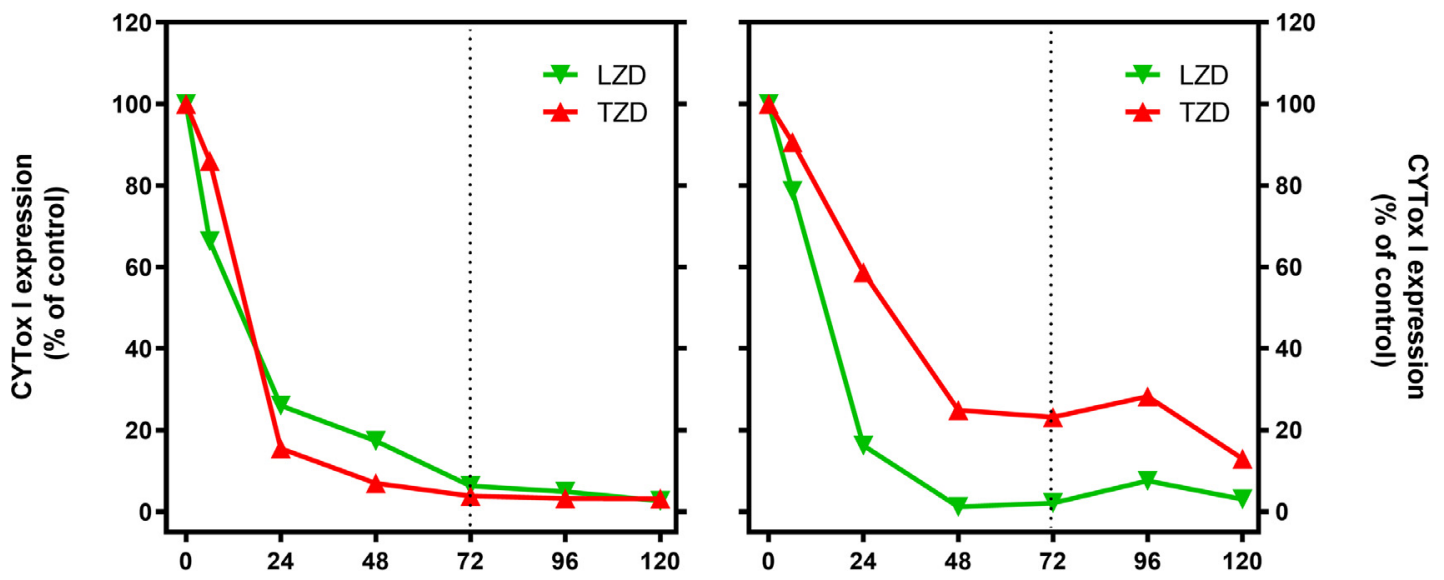

Time (h)

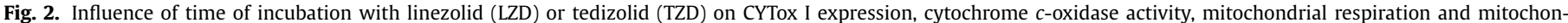

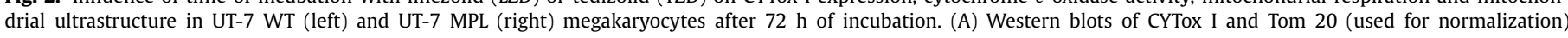

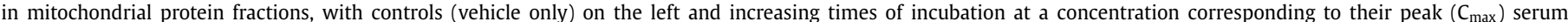

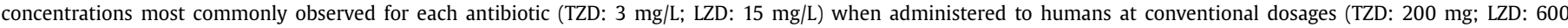

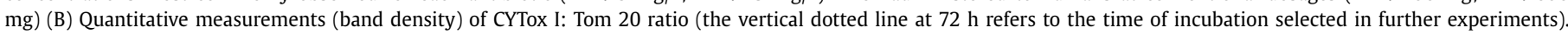

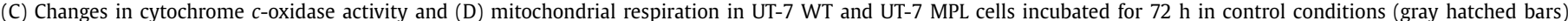

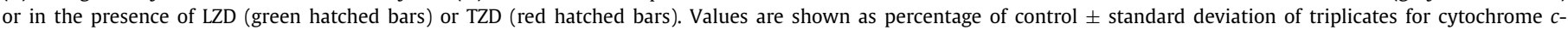

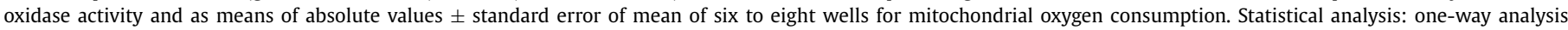

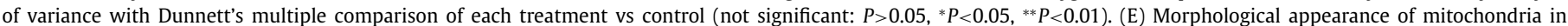

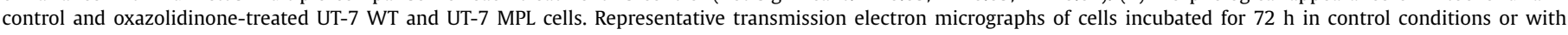
LZD or TZD at the extracellular concentration corresponding to their human total $C_{\max }$. Bars: $1 \mu \mathrm{m}$. 


\section{C. cytochrome c-oxidase activity}

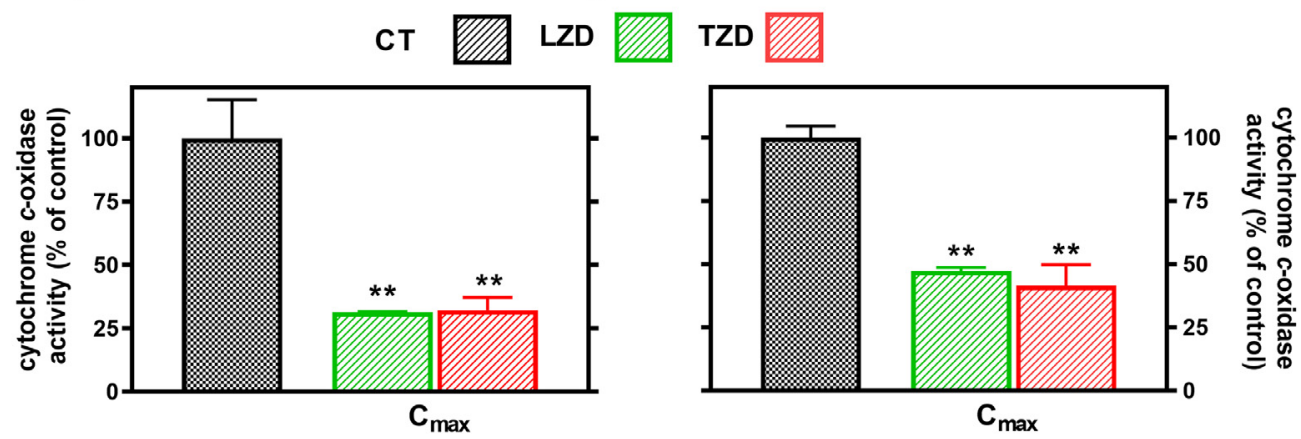

\section{D. mitochondrial oxygen consumption}
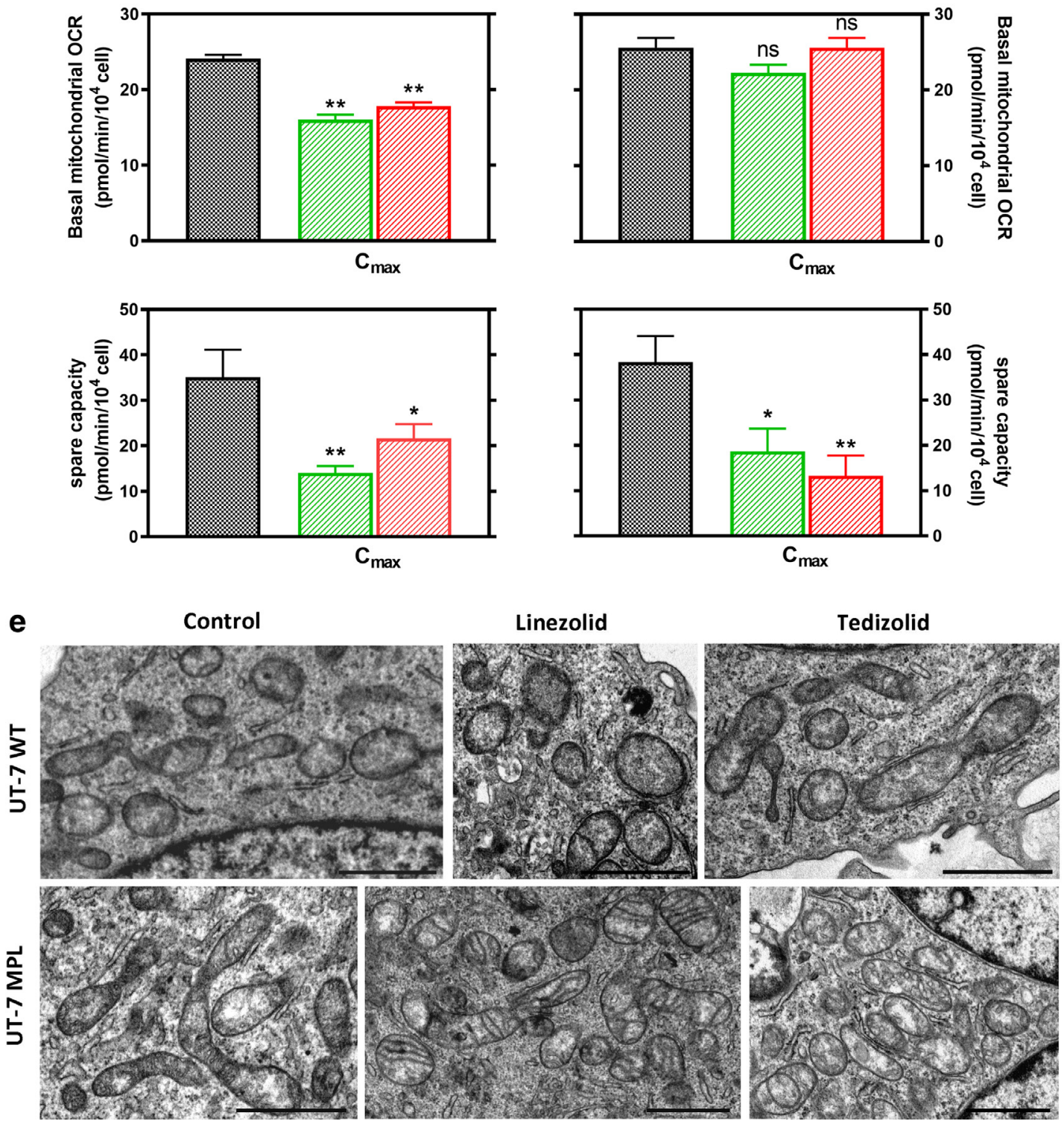

Fig. 2. Continued

expression) and then transferred to drug-free media for up to 144 h. CYTox I expression recovered completely in both cell lines after $72 \mathrm{~h}$ of washout (Fig. 3A,B), but this period of time was insufficient to completely restore cytochrome $c$-oxidase activity in both cell lines. Prolonging the washout period to $144 \mathrm{~h}$ allowed for increased recovery of enzyme activity after linezolid treatment but not after tedizolid treatment (Fig. 3C). This study also tested whether drug washout would allow for recovery of mitochondrial spare capacity, and found that it was variable but only partial in most cases after 3 days (data not shown). 
a

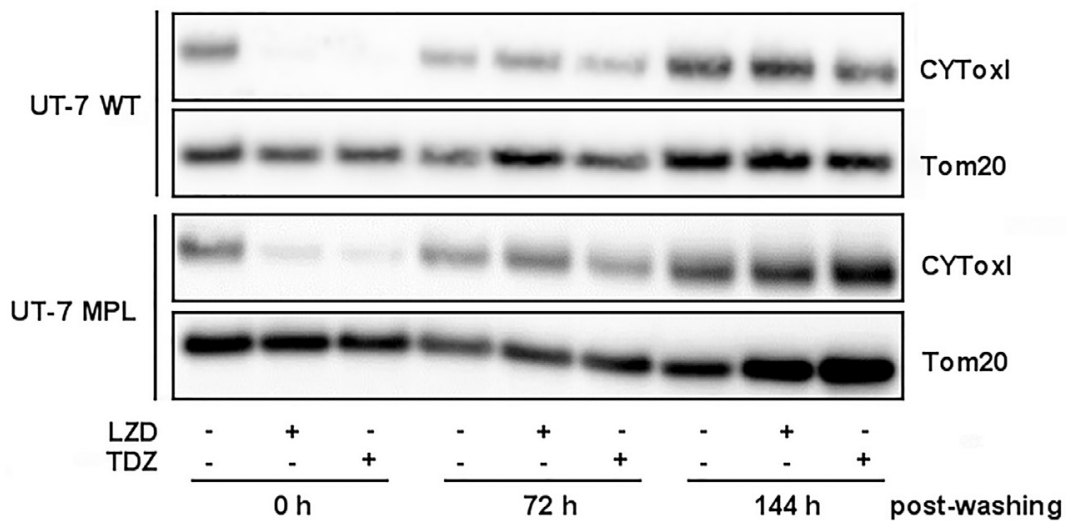

b

UT7-WT
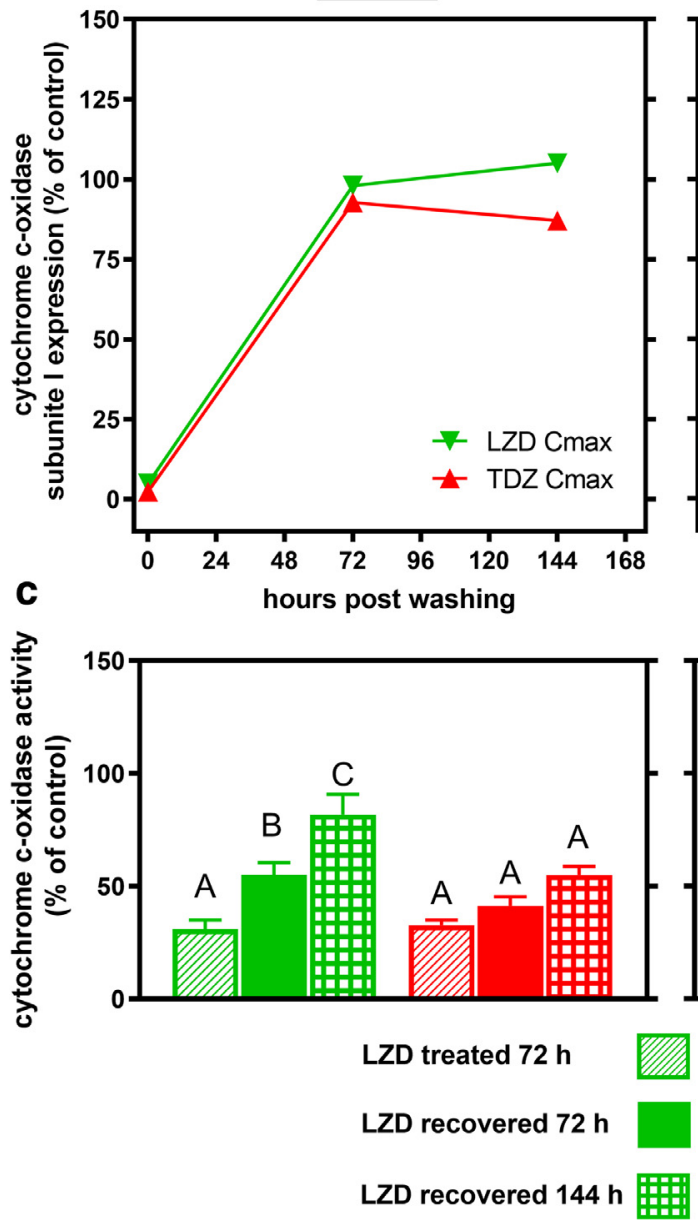

\section{UT7-MPL}
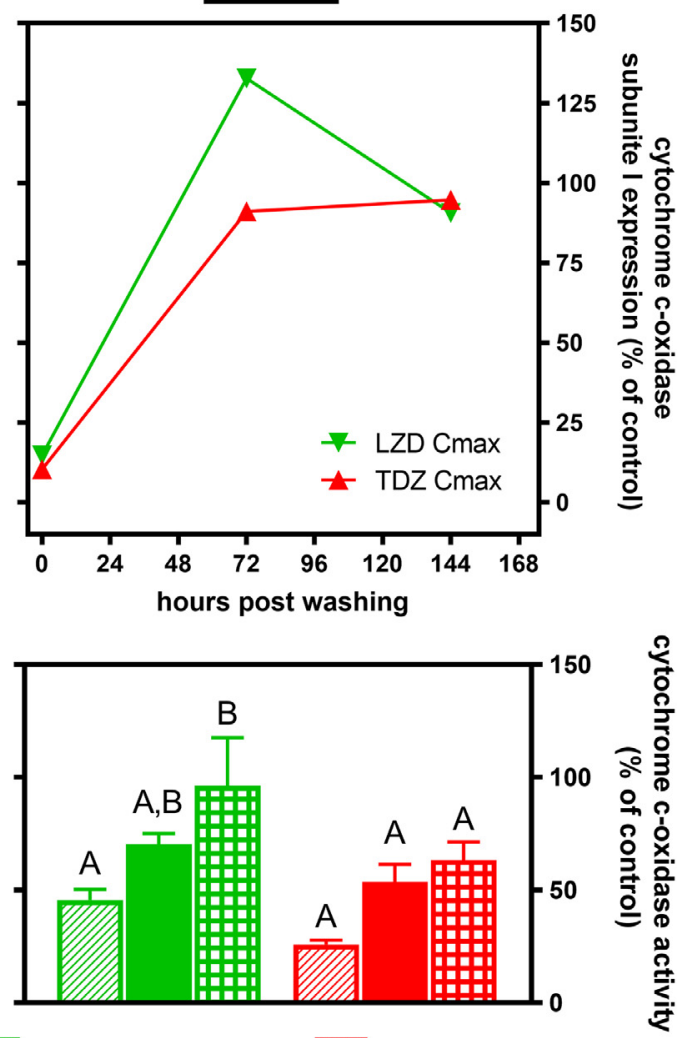

TZD treated $72 \mathrm{~h}$

TZD recoverd $72 \mathrm{~h}$

TZD recoverd $144 \mathrm{~h}$ 曲曲

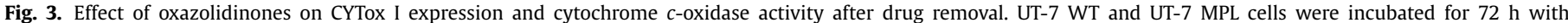

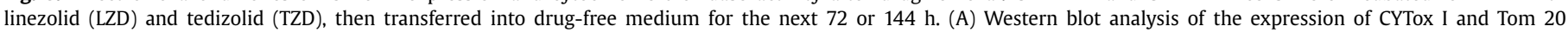

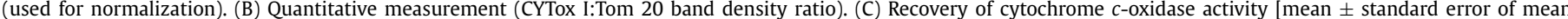

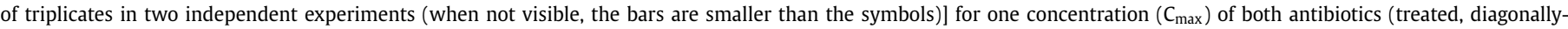

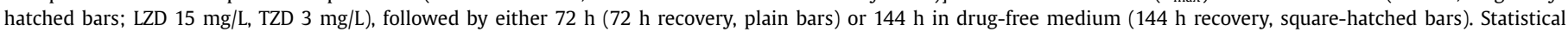

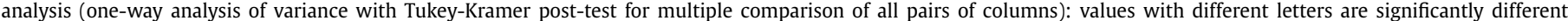
from each other $(P<0.05)$.

\section{Discussion}

Inhibition of mitochondrial protein synthesis by oxazolidinones, due to the existence of common binding sites for oxazolidinones in bacterial and human mitochondrial ribosomes [10], has been reported in both preclinical and clinical studies [8,15]. It has been convincingly associated with impairment of mitochondrial oxidative metabolism in human promyelocytic (HL-60) and monocytic (THP-1) cell lines [9]. This study presents data using cell lines that are more directly related to platelet-generating cells in vivo, namely megakaryoblasts and megakaryocytes. The key observations were that (i) UT-7 cells are as susceptible as other cell lines to the inhibitory effects of oxazolidinones on key mitochondrial parameters, namely CYTox I expression (encoded by 
mitochondrial genome), cytochrome c-oxidase activity and oxidative metabolism; (ii) but that some of these inhibitory effects, especially those caused by tedizolid, are less easily reversed compared with what has been observed with the cell lines examined to date. Interactions between oxazolidinones and bacterial ribosomes are non-covalent and, therefore, should be reversible. In UT7 cells, CYTox I expression was readily reversed in oxazolidinonefree medium, but the recovery of cytochrome c-oxidase activity was slower and incomplete, especially in cells exposed to tedizolid. Of interest, the effects on UT-7 MPL cells, which are more differentiated megakaryoblasts/megakaryocytes and have a maximal TpoR cell-surface expression, were more pronounced than on UT-7 parental cells. Possibly, basal signalling by the TpoR itself might activate pathways that regulate mitochondrial size and components which are relevant for toxicity and recovery in the context of oxazolidinones. It can also be speculated that the perturbations induced by oxazolidinones on mitochondrial structure are so severe that they alter the correct assembly of the cytochrome $c$-oxidase complex. Cytochrome c-oxidase is made of 14 subunits, three of which (CYTox I, II and III) are encoded by the mitochondrial genome. Specific inhibition of their synthesis by oxazolidinones would create an imbalance between all the subunits necessary for cytochrome $c$-oxidase expression and activity [16], resulting in a disassembly of complex IV that may not be easily reversed. Also, it is known that phorbol-myristate-induced differentiation of megakaryocytes is accompanied by a reduction in their mitochondrial activity and alteration of mitochondrial ultrastructure (diminished matrix density, disorganized cristae) [17] reminiscent of what was observed in the present study for UT-7 cell lines when exposed to oxazolidinones. Mitochondrial fragmentation has been shown to occur during megakaryoblast differentiation, probably as a compensatory response to restore adequate mitochondrial function [17]. This may become impossible and result in irreversible loss of function if oxazolidinones are present. An increase in mitochondrial DNA content and RNA expression after linezolid therapy has also been suggested as a compensatory mechanism to prevent further damage [18]. Although calling for further studies, the present data may already identify megakaryocytes as a privileged target for expression of toxicity in vivo. The non-recoverable impairment of their mitochondrial functions would lead to progressive thrombocytopenia, as is observed in patients who then require another full round of megakaryoblast and megakaryocyte formation from haematopoietic precursors/stem cells before correcting for the platelet deficit. The dynamics of platelet formation and destruction would favour negative clinical effects. This is largely in line with the recent pharmacokinetic modelling approaches describing how oxazolidinones interfere with the whole process of megakaryocyte differentiation and platelet release [19].

Finally, tedizolid caused more extensive and sustained mitochondrial alterations than linezolid, even when comparing equitherapeutic concentrations. While this may describe an in-vitro reality (also observed with isolated mitochondria [20]), the clinical situation could be different since the conditions of use in registration clinical trials (dosages [200 mg for tedizolid vs. $600 \mathrm{mg}$ for linezolid] and frequency of administration (once daily for tedizolid vs. twice daily for linezolid) as well as the extent of protein binding of tedizolid and linezolid are quite different (see discussion in [9] and [12]). Thus, true toxicity ranking will require more detailed comparative in-vitro studies, as well as more extensive clinical experience.

\section{Acknowledgements}

The authors wish to thank V. Mohymont, K. Santos Saial and P. Thomas for help in the performance of experiments.
Funding: This work received no specific grant from any funding agency in the public, commercial or not-for-profit sectors, and was covered by the general budget of the participating laboratories.

Competing interests: PMT has received speaker's honoraria from Bayer and research grants from Trius. SNC is the Founder of MyeloPro Research and Diagnostics GmbH, Vienna, Austria and of AlsaTech, Boston, MA, USA. The other authors have no competing interests to declare.

Ethical approval: Not required.

\section{References}

[1] Wilson DN, Schluenzen F, Harms JM, Starosta AL, Connell SR, Fucini P. The oxazolidinone antibiotics perturb the ribosomal peptidyl-transferase center and effect tRNA positioning. Proc Natl Acad Sci USA 2008;105:13339-44.

[2] Chen $\mathrm{KH}$, Huang YT, Liao $\mathrm{CH}$, Sheng WH, Hsueh PR. In vitro activities of tedizolid and linezolid against Gram-positive cocci associated with acute bacterial skin and skin structure infections and pneumonia. Antimicrob Agents Chemother 2015;59:6262-5.

[3] Natsumoto B, Yokota K, Omata F, Furukawa K. Risk factors for linezolid-associated thrombocytopenia in adult patients. Infection 2014;42:1007-12.

[4] Hanai Y, Matsuo K, Ogawa M, Higashi A, Kimura I, Hirayama S, et al. A retrospective study of the risk factors for linezolid-induced thrombocytopenia and anemia. J Infect Chemother 2016;22:536-42.

[5] Nukui Y, Hatakeyama S, Okamoto K, Yamamoto T, Hisaka A, Suzuki H, et al. High plasma linezolid concentration and impaired renal function affect development of linezolid-induced thrombocytopenia. J Antimicrob Chemother 2013;68:2128-33.

[6] Hardalo C, Lodise TP, Bidell M, Flanagan S, De Anda C, Anuskiewicz S, et al. Clinical safety and tolerability of tedizolid phosphate in the treatment of acute bacterial skin and skin structure infections. Expert Opin Drug Saf 2018;17:359-67.

[7] Lee EY, Caffrey AR. Thrombocytopenia with tedizolid and linezolid. Antimicrob Agents Chemother 2017:62 pii:e01453-17.

[8] Nagiec EE, Wu L, Swaney SM, Chosay JG, Ross DE, Brieland JK, et al. Oxazolidinones inhibit cellular proliferation via inhibition of mitochondrial protein synthesis. Antimicrob Agents Chemother 2005;49:3896-902.

[9] Milosevic TV, Payen VL, Sonveaux P, Muccioli GG, Tulkens PM, Van Bambeke F. Mitochondrial Alterations (Inhibition of Mitochondrial Protein Expression, Oxidative Metabolism, and Ultrastructure) Induced by Linezolid and Tedizolid at Clinically Relevant Concentrations in Cultured Human HL-60 Promyelocytes and THP-1 Monocytes. Antimicrob Agents Chemother 2018 Mar;62(3) pii: e01599-17 - PM:29263063.

[10] Leach KL, Swaney SM, Colca JR, McDonald WG, Blinn JR, Thomasco LM, et al. The site of action of oxazolidinone antibiotics in living bacteria and in human mitochondria. Mol Cell 2007;26:393-402.

[11] Im WB, Choi SH, Park JY, Choi SH, Finn J, Yoon SH. Discovery of torezolid as a novel 5-hydroxymethyl-oxazolidinone antibacterial agent. Eur J Med Chem 2011;46:1027-39

[12] Flanagan S, Fang E, Munoz KA, Minassian SL, Prokocimer PG. Single- and multiple-dose pharmacokinetics and absolute bioavailability of tedizolid. Pharmacotherapy 2014:34:891-900.

[13] Komatsu N, Nakauchi H, Miwa A, Ishihara T, Eguchi M, Moroi M, et al. Establishment and characterization of a human leukemic cell line with megakaryocytic features: dependency on granulocyte-macrophage colony-stimulating factor, interleukin 3, or erythropoietin for growth and survival. Cancer Res $1991 ; 51: 341-8$.

[14] Besancenot R, Roos-Weil D, Tonetti C, Abdelouahab H, Lacout C, Pasquier F, et al. JAK2 and MPL protein levels determine TPO-induced megakaryocyte proliferation vs differentiation. Blood 2014;124:2104-15.

[15] De Vriese AS, Coster RV, Smet J, Seneca S, Lovering A, Van Haute LL, et al. Linezolid-induced inhibition of mitochondrial protein synthesis. Clin Infect Dis 2006;42:1111-17.

[16] Kadenbach B, Huttemann M. The subunit composition and function of mammalian cytochrome c oxidase. Mitochondrion 2015;24:64-76.

[17] Huang R, Zhao L, Chen H, Yin RH, Li CY, Zhan YQ et al. Megakaryocytic differentiation of K562 cells induced by PMA reduced the activity of respiratory chain complex IV. PLoS One 2014;9:e96246.

[18] Garrabou G, Soriano A, Pinos T, Casanova-Molla J, Pacheu-Grau D, Moren C, et al. Influence of mitochondrial genetics on the mitochondrial toxicity of linezolid in blood cells and skin nerve fibers. Antimicrob Agents Chemother 2017;61 pii:e00542-17.

[19] Tsuji Y, Holford NHG, Kasai H, Ogami C, Heo YA, Higashi Y, et al. Population pharmacokinetics and pharmacodynamics of linezolid-induced thrombocytopenia in hospitalized patients. Br J Clin Pharmacol 2017;83:1758-72.

[20] Flanagan S, McKee EE, Das D, Tulkens PM, Hosako H, Fiedler-Kelly J, et al. Nonclinical and pharmacokinetic assessments to evaluate the potential of tedizolid and linezolid to affect mitochondrial function. Antimicrob Agents Chemother 2015;59:178-85. 